\title{
Niclosamide inhibits the proliferation of human osteosarcoma cell lines by inducing apoptosis and cell cycle arrest
}

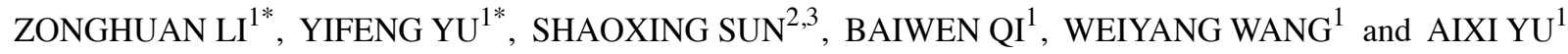 \\ Departments of ${ }^{1}$ Orthopedics, ${ }^{2}$ Radiation and Medical Oncology, Zhongnan Hospital of Wuhan University; \\ ${ }^{3}$ Hubei Key Laboratory of Tumor Biological Behaviors, Zhongnan Hospital \\ of Wuhan University, Wuhan, Hubei 430071, P.R. China
}

Received October 20, 2014; Accepted January 12, 2015

DOI: $10.3892 /$ or.2015.3766

\begin{abstract}
Niclosamide, used as an antihelminthic, has demonstrated some properties of anticancer effects. However, its role in osteosarcoma remains to be determined. The aim of this study was to determine the effect of niclosamide on human osteosarcoma cell lines. The human MG-63 and U2OS osteosarcoma cell lines were treated with different concentrations of niclosamide. The cell inhibitory rate was calculated by CCK- 8 assay. Cell cycle was detected by flow cytometry. Cell apoptosis was determined by Hoechst 33324 staining, flow cytometry and fluorescence microscope, respectively. The expression of bcl-2, bax and pro-caspase- 3 were measured by western blotting. Niclosamide exerted an inhibitory effect on the two cell lines in a time- and dose-dependent manner. Niclosamide was found to induce the arrest of $\mathrm{S}$ and G2/M phase in U2OS cells and G2/M in MG-63 cells. Moreover, niclosamide induced apoptosis in MG-63 and U2OS cells. The bax/bcl-2 ratio increased while the expression of pro-caspase- 3 decreased significantly in the two cell lines. The results indicated that niclosamide inhibits proliferation, and induces apoptosis and cell cycle arrest in human osteosarcoma cell lines.
\end{abstract}

\section{Introduction}

Osteosarcoma is the most frequent primary malignant bone tumor in young adolescents and children (1). Currently, patients with osteosarcoma are evaluated and treated using multidisciplinary treatments including surgical resection of the lesions, radiotherapy, adjuvant chemotherapy and neoadjuvant chemotherapy (1). Combination of a variety of chemothera-

Correspondence to: Dr Aixi Yu, Department of Orthopedics, Zhongnan Hospital of Wuhan University, No. 169 Donghu Road, Wuchang, Wuhan, Hubei 430071, P.R. China

E-mail: yuaixi666@hotmail.com

${ }^{*}$ Contributed equally

Key words: niclosamide, osteosarcoma, proliferation, apoptosis peutic drugs is usually recommended during the process of chemotherapy. However, traditional chemotherapeutic drugs result in greater renal, cardiac and auditory dysfunction toxicity, and $30-40 \%$ of patients with osteosarcoma still have a poor response (2). Therefore, drug development is important for the treatment of osteosarcoma.

Niclosamide, a type of salicylamide derivative, is an antihelminthic drug that has been used for approximately 50 years for tapeworm infections $(3,4)$. In the past few years, several groups have independently found that niclosamide is a potential anticancer drug. In 2011, Sack et al (5) screened 1,280 types of pharmaceutically active compounds using high-throughput screening technology and found niclosamide was able to inhibit colon cancer metastasis. Recently, Wieland et al (6) found that niclosamide is cytotoxic, inhibits cell migration and enhances the effects of chemotherapeutic drugs in glioma cells. Another study (7) found that niclosamide inhibits the growth and induces apoptosis of acute myeloid leukemia cells in vitro and in nude mice, while cells from normal bone marrow were spared.

Niclosamide has been confirmed as a potential anticancer drug. Previous studies found that niclosamide exerted inhibitory effects on tumors of epithelial origin. However, its role in mesenchymal-derived tumors (e.g., osteosarcoma) remains to be determined. The aim of this study was to investigate the role of niclosamide in human osteosarcoma cells.

\section{Materials and methods}

Materials and chemicals. Niclosamide was purchased from Wuhan Shengtianyu Biotech (Wuhan, China). It was dissolved in dimethyl sulfoxide (DMSO) and stored at $4^{\circ} \mathrm{C}$ at the concentration of $2 \mathrm{mM}$. The cell counting kit-8 (CCK-8) was purchased from Dojindo Molecular Technologies (Kumamoto, Japan). Hoechst 33342 was obtained from Bioyear Biotech (Wuhan, China). The Annexin V-FITC apoptotic detection kit was purchased from BestBio Biotech (Shanghai, China). Propidium iodide (PI)/RNase was purchased from Tianjin Sungene Biotech Co., Ltd. (Tianjin, China). The primary antibodies against bcl-2, bax, and pro-caspase-3 and the secondary antibodies were purchased from Santa Cruz Biotechnology, Inc. (Santa Cruz, CA, USA). All the reagents were stored at $4^{\circ} \mathrm{C}$ prior to use. 

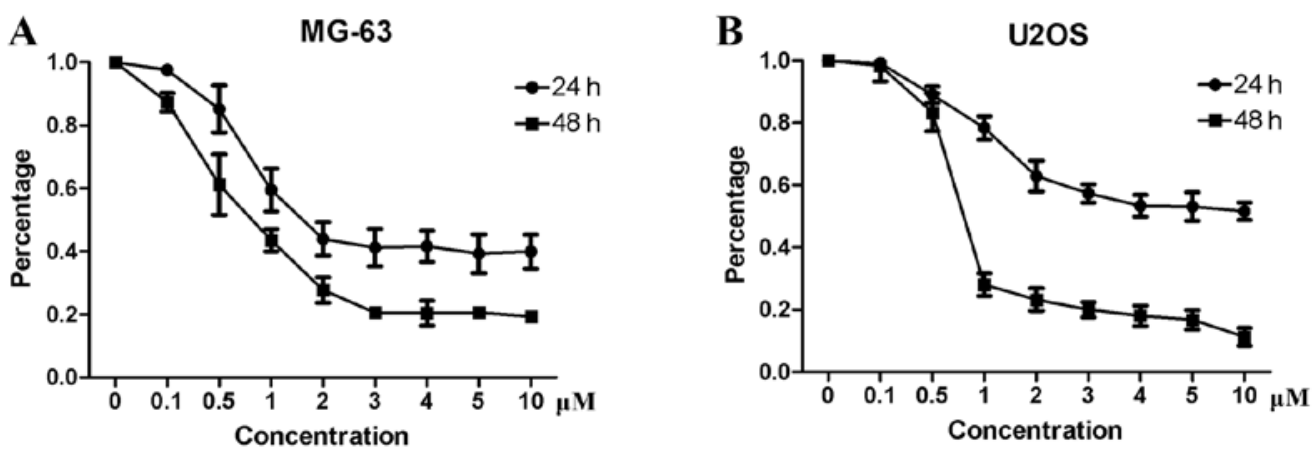

Figure 1. Inhibitory effects of niclosamide on the proliferation of MG-63 (A) and U2OS (B) cells following treatment for 24 and 48 h. The two cell lines were inhibited by niclosamide in a time- and dose-dependent manner.

Cell culture. The human MG-63 and U2OS osteosarcoma cell lines were obtained from the China Center for Type Culture Collection (Wuhan, China). The cells were cultured in DMEM medium with high glucose (HyClone, Logan, UT, USA) containing $10 \%$ fetal bovine serum (Gibco, Carlsbad, CA, USA). The cells were incubated in a constant environment at $37^{\circ} \mathrm{C}$ with $95 \%$ air and $5 \% \mathrm{CO}_{2}$. Medium renewal was performed every 2-3 days. The cells were subcultured when $90 \%$ confluence was reached.

CCK- 8 assay. Cells $\left(5 \times 10^{3}\right)$ were seeded in 96 -well plates and cultured overnight. Niclosamide $(0,0.1,0.5,1,2,3,4,5$ and $10 \mu \mathrm{M}$ ) was added and incubated for 24 and $48 \mathrm{~h}$, respectively. The medium was replaced with $100 \mu \mathrm{l}$ fresh medium and $10 \mu \mathrm{l}$ CCK-8 solution. The cells were incubated for an additional $4 \mathrm{~h}$ and the absorbance was measured at $450 \mathrm{~nm}$ by an Enspire microplate reader (PerkinElmer, Inc., Waltham, MA, USA). The cell viability and $\mathrm{IC}_{50}$ values were calculated.

Hoechst 33342 staining. Cells $\left(5 \times 10^{5}\right)$ were transferred in 6-well plates and cultured overnight. Niclosamide $(0,1,2$ and $5 \mu \mathrm{M})$ was added and incubated for $24 \mathrm{~h}$. The medium was replaced

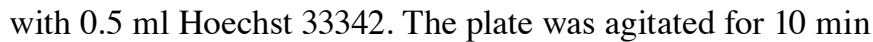
in the dark and analyzed using a fluorescence microscope (Olympus BX51, Olympus, Tokyo, Japan).

Cell cycle analysis. After various treatments, the cells were collected and fixed with cold $70 \%$ ethanol at room temperature (RT) for $1 \mathrm{~h}$. The cells were then washed twice with PBS and stained with $0.5 \mathrm{ml} \mathrm{PI/RNase}$ at RT for $30 \mathrm{~min}$. The samples were analyzed by flow cytometry (FC 500, Beckman Coulter, Brea, CA, USA; BD FACSAria III, Becton Dickinson, Franklin Lakes, NJ, USA).

Annexin V-FITC/PI double staining. Following treatment with various concentrations of niclosamide, the cells were collected and washed twice with PBS. Annexin V binding buffer (400 $\mu \mathrm{l})$ and Annexin V-FITC (5 $\mu \mathrm{l})$ were added. The cells were homogenized and incubated in the dark at $4^{\circ} \mathrm{C}$ for $15 \mathrm{~min}$. PI $(10 \mu \mathrm{l})$ was added and incubated for $5 \mathrm{~min}$. The samples were analyzed by flow cytometry (FC 500, Beckman Coulter).

After various treatments, the medium was removed. Then, $400 \mu 1$ Annexin V binding buffer, $5 \mu 1$ Annexin V-FITC and
$10 \mu 1$ PI solution, respectively, were added. The cells were incubated at RT in the dark for 30 min and analyzed using a fluorescence microscope (Olympus BX51, Olympus).

Western blotting. Cells were similarly treated with various concentrations of niclosamide for $24 \mathrm{~h}$, lysed, collected and centrifuged at $12,000 \mathrm{x} \mathrm{g}$ at $4^{\circ} \mathrm{C}$ for $30 \mathrm{~min}$. The supernatants were obtained and protein concentration was determined by BCA assay.

An equivalent amount of proteins was then separated by SDS-polyacrylamide gel and transferred onto PVDF membranes. The membranes were incubated with $5 \%$ skimmed milk, followed by incubation with primary antibodies overnight at $4^{\circ} \mathrm{C}$ and secondary antibody at RT for $2 \mathrm{~h}$. The immunoblots were developed and visualized with a Supersignal West Pico Assay kit (Thermo Fisher Scientific, Inc., Rockford, IL, USA) and autoradiography film.

Statistical analysis. Data were presented as mean $(\mathrm{M}) \pm$ standard deviation (SD). The Student's t-test was applied to analyze the differences between each niclosamide concentration and the control. $\mathrm{P}<0.05$ was considered significantly different.

\section{Results}

Niclosamide inhibits the proliferation of MG-63 and U2OS. To determine the inhibitory effect of niclosamide on osteosarcoma cells, a CCK-8 assay was performed. The results showed that niclosamide inhibited the proliferation of MG-63 (Fig. 1A) and U2OS (Fig. 1B) in a time- and dose-dependent manner. The $\mathrm{IC}_{50}$ values for MG-63 and U2OS were 1.5 and $5 \mu \mathrm{M}$, respectively.

Niclosamide induces cell cycle arrest of MG-63 and U2OS. Cell cycle analysis was performed with PI staining by flow cytometry. After MG-63 cells were treated by niclosamide for $24 \mathrm{~h}$, the number of cells in the G1 phase decreased gradually, while the number of cells increased in G2 and S phase. For U2OS cells, the cell cycle was mainly arrested in G2 phase. The dose-dependent effects were obvious, especially in U2OS cells (Fig. 2).

Niclosamide induces apoptosis of MG-63 and U2OS. To determine whether niclosamide induces apoptosis of MG-63 

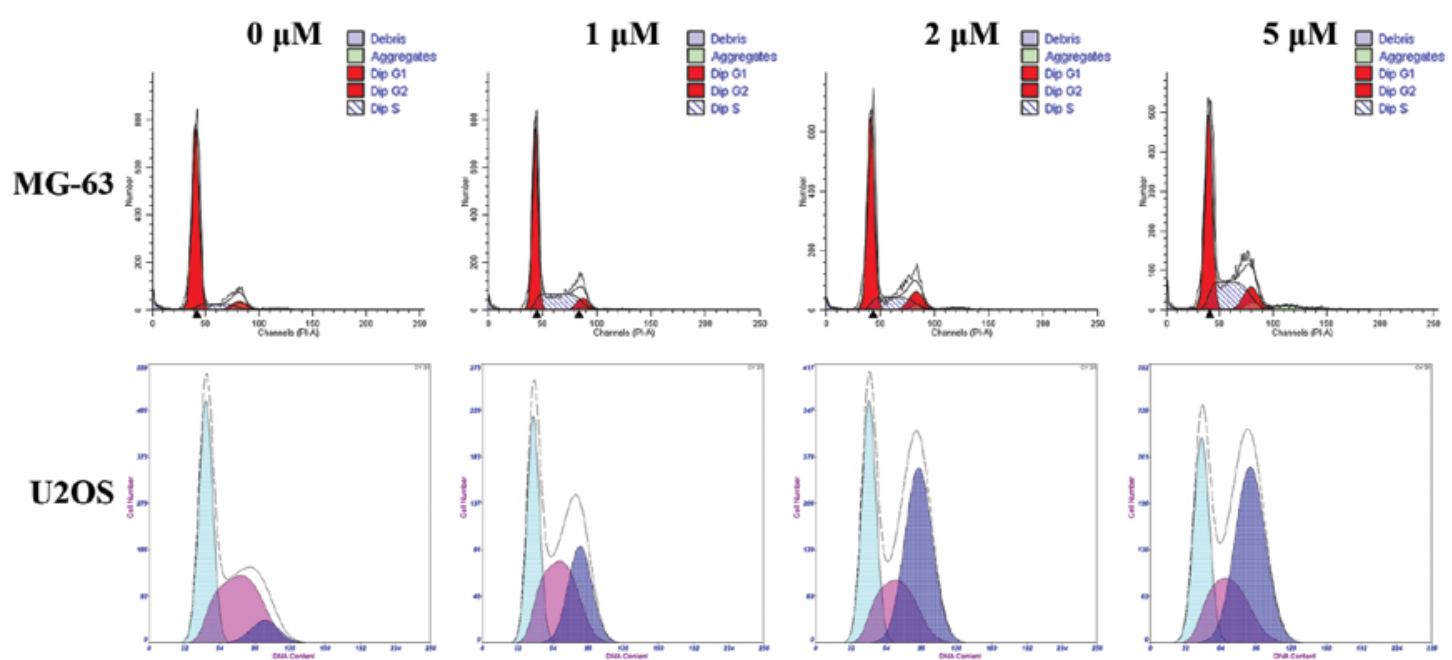

MG-63

U2OS
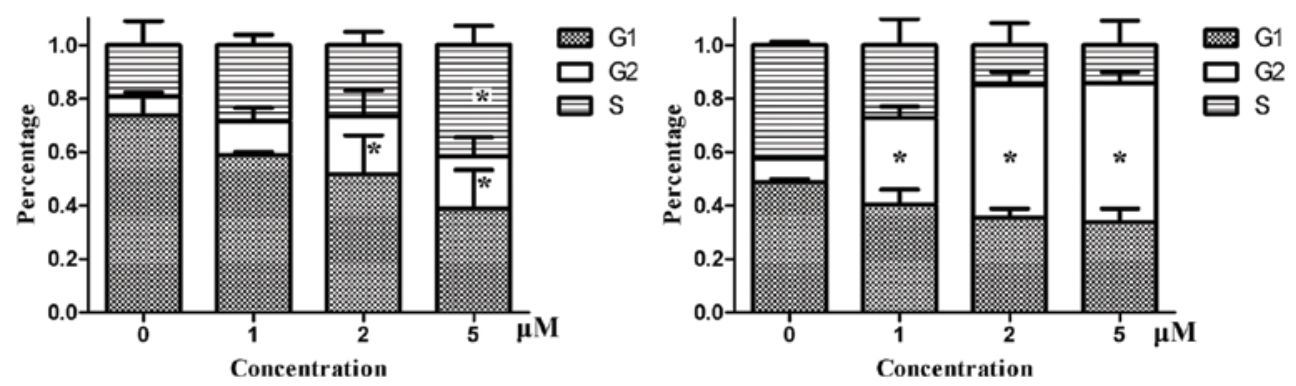

Figure 2. Cell cycle analysis of MG-63 and U2OS cells following treatment with various concentrations of niclosamide for $24 \mathrm{~h}$. MG- 63 cells were arrested in G2 and S phase, while U2OS cells were arrested in G2 phase. The untreated cells were used as the control. "P<0.05.
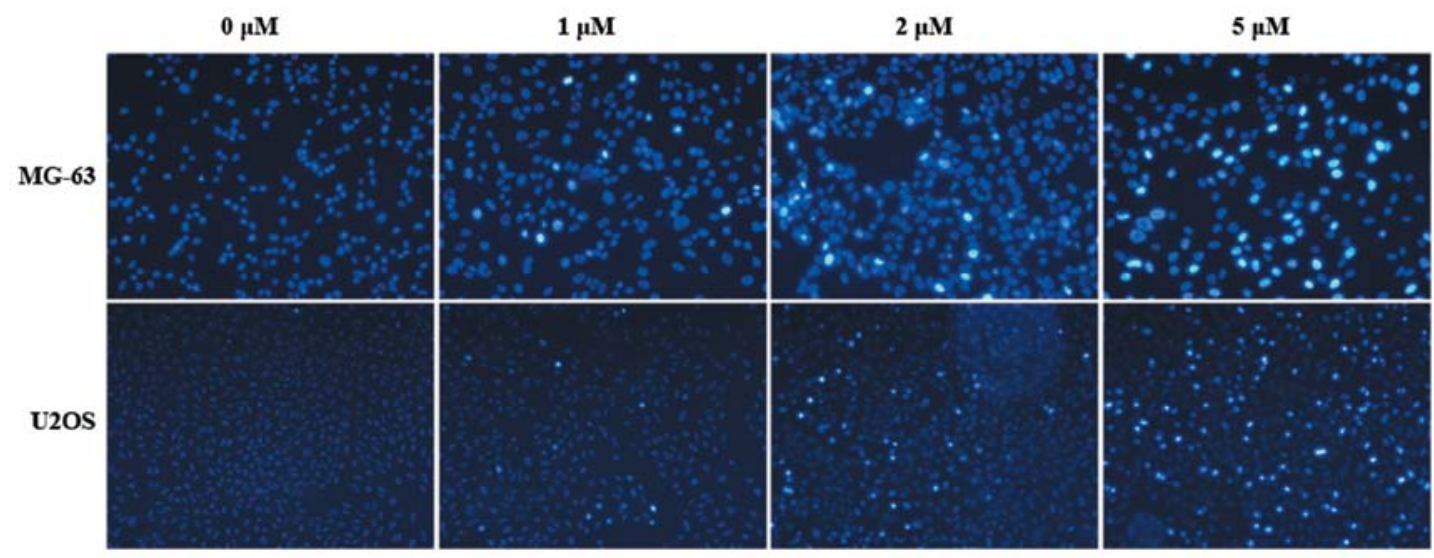

Figure 3. Hoechst 33342 staining of MG-63 and U2OS cells following treatment with various concentrations of niclosamide for $24 \mathrm{~h}$. Cell nuclei dyed bright blue or white were considered as apoptotic cells. No apoptotic cells were observed in the controlled group. In the experimental group, the apoptotic cells were evident and the number of apoptotic cells increased in a dose-dependent manner. The untreated cells were used as the control.

and U2OS cells, Hoechst staining, Annexin V FITC/PI staining and western blotting were performed.

As shown in Fig. 3, the cell nuclei were dyed blue, while the nuclei of apoptotic cells were dyed bright blue or even white. The results showed that the number of apoptotic cells increased gradually in a dose-dependent manner. Niclosamide therefore induced the apoptosis of MG-63 and U2OS cells.

Fig. 4 shows the flow cytometry results of Annexin V-FITC/ PI double staining. Niclosamide induced both early and late apoptosis of MG63 and U2OS cells, which was more evident in U2OS cells.
The two cell lines were observed with a fluorescence microscope after double staining (Fig. 5). Under the light field, the appearance of cells was more sparse in the treatment groups. When the cells were observed with blue/green spectral filters, the number of apoptotic cells significantly increased in a dose-dependent manner.

The expression of bcl-2, bax and pro-caspase-3 was examined by western blotting (Fig. 6). The results showed that pro-caspase-3 decreased significantly, which indicated the activation of apoptosis. The expression of bcl- 2 decreased and an increased expression of bax was observed. The bax/bcl-2 

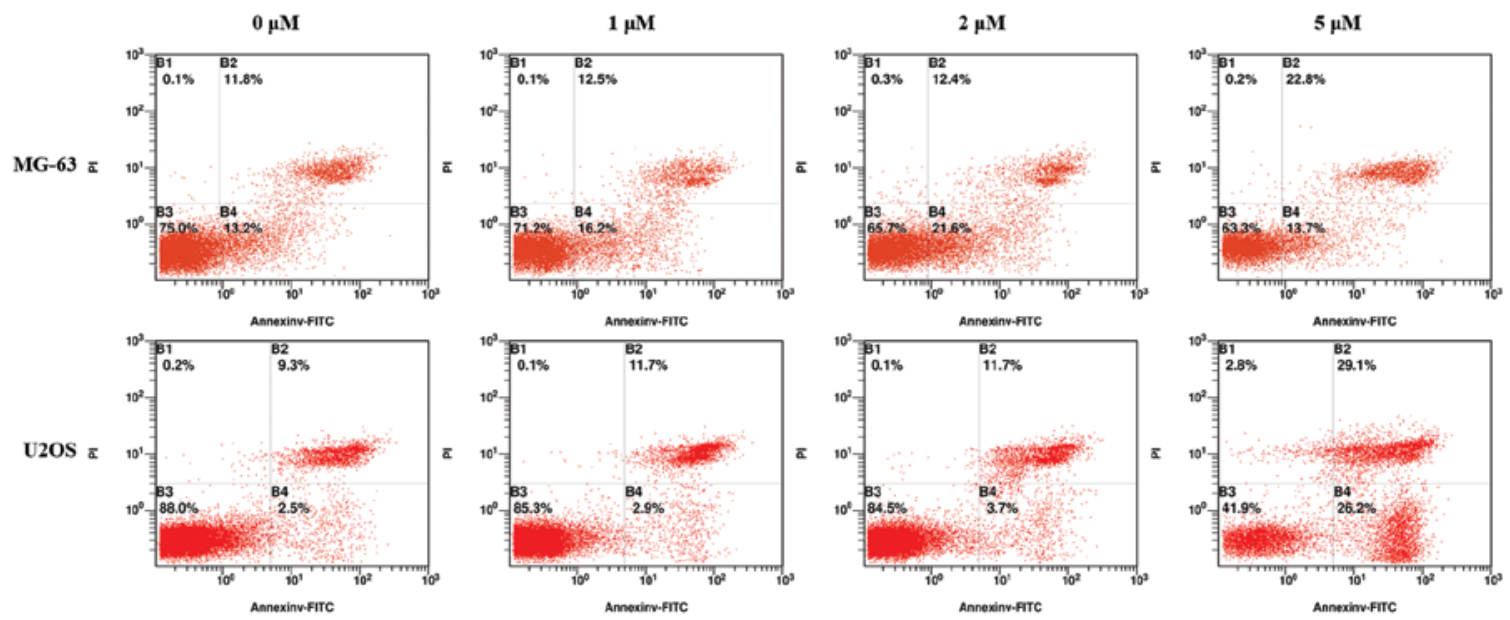

MG-63
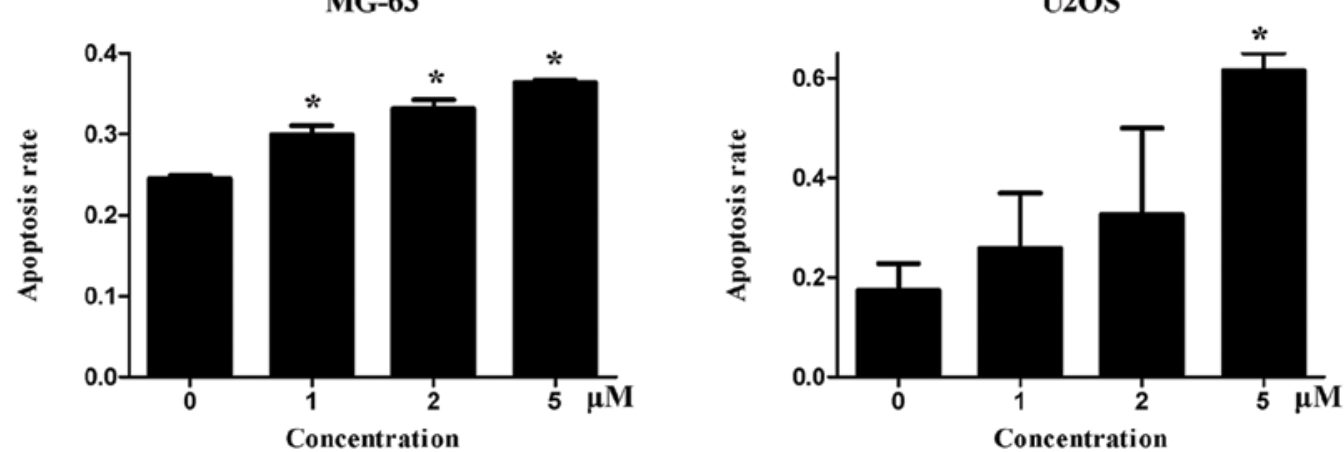

Figure 4. Cell apoptosis of MG-63 (upper panels) and U2OS (lower panels) cells following treatment with various concentrations of niclosamide for $24 \mathrm{~h}$. The apoptotic rate increased significantly, especially in U2OS cells. The untreated cells were used as the control. " $\mathrm{P}<0.05$.

ratio increased following treatment with niclosamide, which further confirmed that niclosamide inhibited cell growth by inducing apoptosis.

\section{Discussion}

Niclosamide, an antitapeworm drug, has been FDA approved for almost 50 years $(3,4)$. In recent years, it has been suggested that niclosamide inhibited cancer growth and metastasis in epithelium-derived tumors, including prostate $(8)$, lung $(9,10)$, head and neck (11), and ovarian cancer $(12,13)$. However, its effects on mesenchymal tumors (e.g., osteosarcoma) are unclear. Thus, we performed this study to determine this issue. The results of the present study demonstrate that niclosamide inhibits proliferation, induces cell cycle arrest and apoptosis in human osteosarcoma cells in a time- and dose-dependent manner.

In the development of new chemotherapeutic agents, issues that need to be addressed concern evaluation of the efficacy and safety of such drugs. Niclosamide, which has been approved by FDA for 50 years $(3,4)$, is safe for oral ingestion. Furthermore, niclosamide induces apoptosis in acute myelocytic leukemia cells but spares cells from normal bone marrow (7). Thus, niclosamide is generally regarded as safe and efficient in many tumors, including colon cancer $(5,14)$, breast cancer $(3,4)$, and prostate cancer $(8)$. In the present study, we evaluated the efficacy of niclosamide on human cancer cells in vitro. The results show that it is a potential anticancer drug for osteosarcoma.
Previous studies revealed that niclosamide induces growth inhibition and cell apoptosis by cell cycle arrest $(15,16)$. However, the cell cycle arrest of various cancer cells by niclosamide is not phase-specific. Niclosamide induces different cell cycle distribution in different cancer cells. For example, the flow cytometric analysis showed that niclosamide induced S-phase arrest in human erythroleukemia K562 cells (17). However, the cell population in G1 phase transiently increased following treatment with niclosamide in primary human glioblastoma cells (6). In Du145 prostate cancer cells, G0/G1-phase arrest has been observed (18). In this study, cell cycle analysis was performed in two different osteosarcoma cell lines, MG-63 and U2OS. The cell cycle distribution was found to be different from the results of previous studies. Niclosamide induces G2/S arrest in MG-63 cells, but G2 phase arrest in U2OS cells. Thus, niclosamide is a cancer cellspecific cell-cycle inhibitor.

Bcl-2, an antiapoptotic protein, inhibits a number of programs in cell apoptosis (19). Bax is identified as a bcl2 -interacting protein that inhibits the activity of bcl-2 and promotes apoptotic cell death (20). An increased bcl-2/bax ratio is usually detected when apoptotic death occurs (21). In the present study, we found that niclosamide induces apoptosis of MG-63 and U2OS cells by decreasing the bcl-2/bax ratio, a finding that was consistent with a previous study (4). Caspase- 3 plays a key role in the process of apoptosis $(22,23)$. It is associated with morphological changes of apoptosis and apoptotic body formation (22). When apoptosis occurs, caspase- 3 is activated and cleaved into two subunits (23). The 

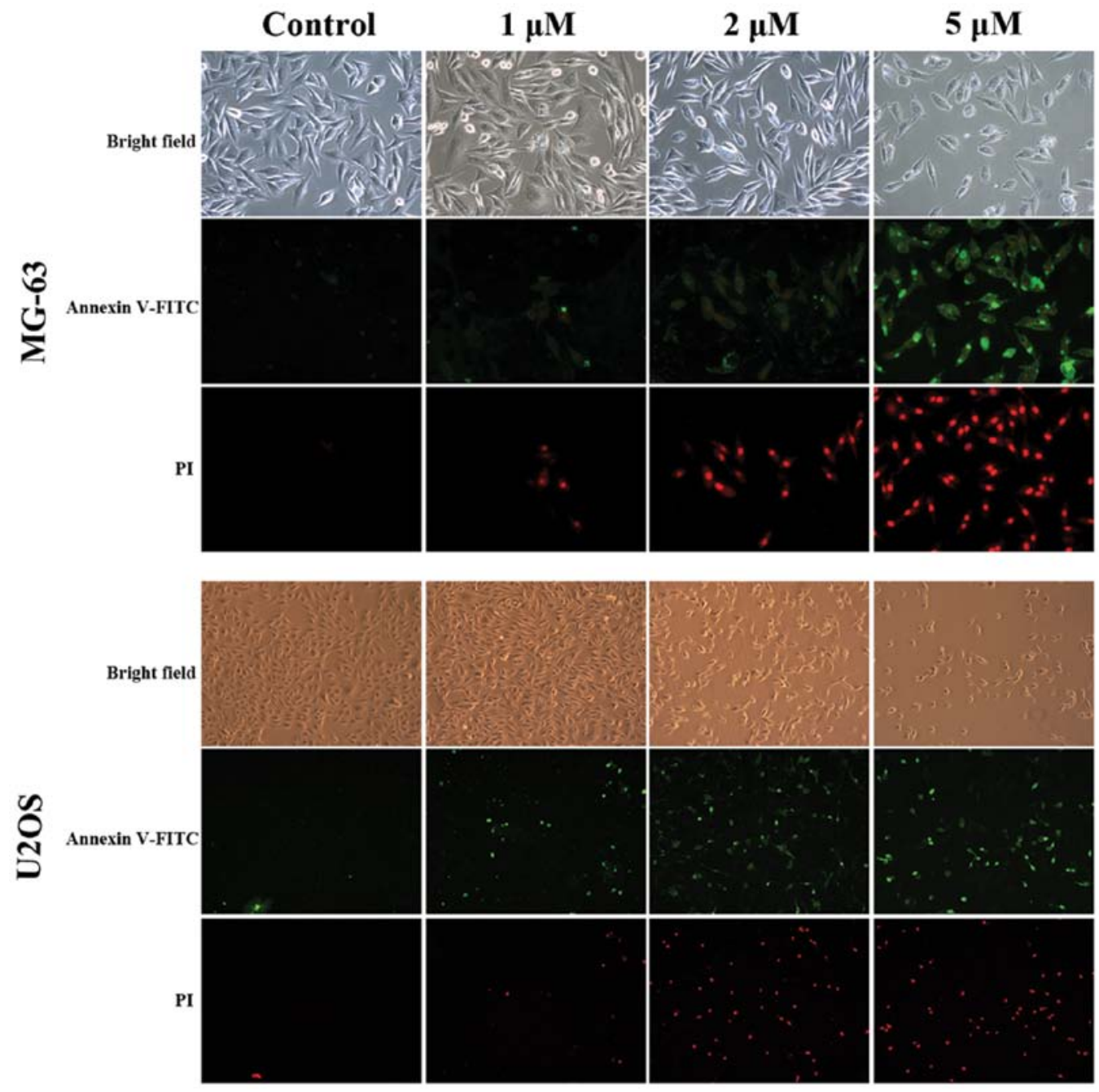

Figure 5. Fluorescent micrographs of apoptotic cells after staining with Annexin V-FITC/PI. Apoptotic cells were sparse in the untreated cells. The apoptotic cells with green membrane and red nucleus were evident in the treated cells $(1,2$, and $5 \mu \mathrm{M})$. The untreated cells were used as the control.

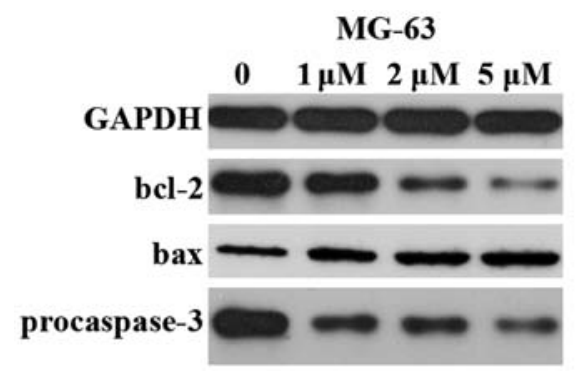

U2OS

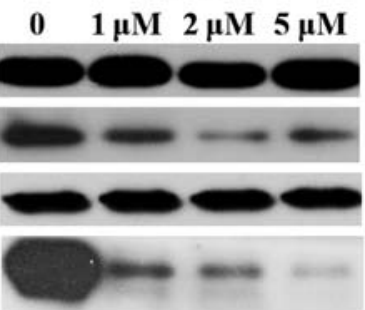

Figure 6. Effects of niclosamide on bcl-2, bax and pro-caspase-3 expression as detected using western blotting. The cells were treated with niclosamide for $24 \mathrm{~h}$. GAPDH was used as an internal reference.

decreased expression of procaspase-3 in our study further confirmed that niclosamide induces apoptosis in human osteosarcoma cells.

To the best of our knowledge, this is a pilot study and some issues deserve mentioned. First, apoptosis is induced by niclosamide but the major mechanism is not clear. It is well known that niclosamide inhibits oxidative phosphorylation in the mitochondria of cestodes. Niclosamide affects cancer cells in several different pathways, including Wnt/ $\beta$ catenin $(24,25)$, mTORC1 signaling $(26,27)$, STAT 3 (10), and
ROS (28). Further studies are required to determine the exact mechanism in osteosarcoma cells. Second, it has been found that niclosamide induces apoptosis in cancer cells but spares normal cells (7). The reason for this difference in apoptosis induction between the normal cells and cancer cells remains to be identified through a more comprehensive investigation of the apoptotic mechanism. Furthermore, the present study was performed in vitro but not in vivo. A limitation of this is that certain chemicals show excellent anticancer effects in vitro but not in vivo. Animal studies and clinical studies are 
therefore needed before niclosamide is proven as an effective anticancer drug.

In conclusion, the present study has demonstrated that niclosamide inhibits proliferation, induces cell cycle arrest and apoptosis of human osteosarcoma cells. Niclosamide may be developed into a novel and potential chemotherapeutic drug for osteosarcoma.

\section{Acknowledgements}

This study was supported by Hubei Province's Outstanding Medical Academic Leader Program.

\section{References}

1. Biermann JS, Adkins DR, Agulnik M, et al: Bone cancer. J Natl Compr Canc Netw 11: 688-723, 2013.

2. Hogendoorn PC; ESMO/EUROBONET Working Group, Athanasou N, Bielack S, et al: Bone sarcomas: ESMO Clinical Practice Guidelines for diagnosis, treatment and follow-up. Ann Oncol 21 (Suppl 5): v204-v213, 2010.

3. Londono-Joshi AI, Arend RC, Aristizabal L, et al: Effect of niclosamide on basal-like breast cancers. Mol Cancer Ther 13: 800-811, 2014

4. Ye T, Xiong Y, Yan Y, et al: The anthelmintic drug niclosamide induces apoptosis, impairs metastasis and reduces immunosuppressive cells in breast cancer model. PLoS One 9: e85887, 2014.

5. Sack U, Walther W, Scudiero D, et al: Novel effect of antihelminthic Niclosamide on S100A4-mediated metastatic progression in colon cancer. J Natl Cancer Inst 103: 1018-1036, 2011.

6. Wieland A, Trageser D, Gogolok S, et al: Anticancer effects of niclosamide in human glioblastoma. Clin Cancer Res 19: 4124-4136, 2013.

7. Jin Y, Lu Z, Ding K, et al: Antineoplastic mechanisms of niclosamide in acute myelogenous leukemia stem cells: inactivation of the NF-kappaB pathway and generation of reactive oxygen species. Cancer Res 70: 2516-2527, 2010.

8. Liu C, Lou W, Zhu Y, et al: Niclosamide inhibits androgen receptor variants expression and overcomes enzalutamide resistance in castration-resistant prostate cancer. Clin Cancer Res 20: 3198-210, 2014.

9. Li R, Hu Z, Sun SY, et al: Niclosamide overcomes acquired resistance to erlotinib through suppression of STAT3 in non-small cell lung cancer. Mol Cancer Ther 12: 2200-2212, 2013.

10. You S, Li R, Park D, et al: Disruption of STAT3 by niclosamide reverses radioresistance of human lung cancer. Mol Cancer Ther 13: 606-616, 2014.

11. Li R, You S, Hu Z, et al: Inhibition of STAT3 by niclosamide synergizes with erlotinib against head and neck cancer. PLoS One 8: e74670, 2013.
12. Yo YT, Lin YW, Wang YC, et al: Growth inhibition of ovarian tumor-initiating cells by niclosamide. Mol Cancer Ther 11: 1703-1712, 2012 .

13. Arend RC, Londoño-Joshi AI, Samant RS, et al: Inhibition of Wnt/ $\beta$-catenin pathway by niclosamide: A therapeutic target for ovarian cancer. Gynecol Oncol 34: 112-120, 2014.

14. Chen W, Chen M and Barak LS: Development of small molecules targeting the Wnt pathway for the treatment of colon cancer: a high-throughput screening approach. Am J Physiol Gastrointest Liver Physiol 299: G293-G300, 2010.

15. Li Y, Li PK, Roberts MJ, et al: Multi-targeted therapy of cancer by niclosamide: A new application for an old drug. Cancer Lett 349: 8-14, 2014.

16. Pan JX, Ding K and Wang CY: Niclosamide, an old antihelminthic agent, demonstrates antitumor activity by blocking multiple signaling pathways of cancer stem cells. Chin J Cancer 31: 178-184, 2012.

17. Wang $\mathrm{AM}, \mathrm{Ku} \mathrm{HH}$, Liang YC, et al: The autonomous notch signal pathway is activated by baicalin and baicalein but is suppressed by niclosamide in K562 cells. J Cell Biochem 106: 682-692, 2009.

18. Ren X, Duan L, He Q, et al: Identification of niclosamide as a new small-molecule inhibitor of the STAT3 signaling pathway. ACS Med Chem Lett 1: 454-459, 2010.

19. Reed JC: Proapoptotic multidomain Bcl-2/Bax-family proteins: mechanisms, physiological roles, and therapeutic opportunities. Cell Death Differ 13: 1378-1386, 2006.

20. Oltvai ZN, Milliman CL and Korsmeyer SJ: Bcl-2 heterodimerizes in vivo with a conserved homolog, Bax, that accelerates programmed cell death. Cell 74: 609-619, 1993.

21. Korsmeyer SJ, Shutter JR, Veis DJ, et al: Bcl-2/Bax: a rheostat that regulates an anti-oxidant pathway and cell death. Semin Cancer Biol 4: 327-332, 1993.

22. Porter AG and Janicke RU: Emerging roles of caspase-3 in apoptosis. Cell Death Differ 6: 99-104, 1999.

23. Cohen GM: Caspases: the executioners of apoptosis. Biochem J 326: 1-16, 1997.

24. Lu W, Lin C, Roberts MJ, et al: Niclosamide suppresses cancer cell growth by inducing Wnt co-receptor LRP6 degradation and inhibiting the Wnt/ $\beta$-catenin pathway. PLoS One 6: e29290, 2011.

25. Tomizawa M, Shinozaki F, Motoyoshi Y, et al: Niclosamide suppresses hepatoma cell proliferation via the Wnt pathway. Onco Targets Ther 6: 1685-1693, 2013.

26. Fonseca BD, Diering GH, Bidinosti MA, et al: Structure-activity analysis of niclosamide reveals potential role for cytoplasmic $\mathrm{pH}$ in control of mammalian target of rapamycin complex 1 (mTORC1) signaling. J Biol Chem 287: 17530-17545, 2012.

27. Balgi AD, Fonseca BD, Donohue E, et al: Screen for chemical modulators of autophagy reveals novel therapeutic inhibitors of mTORC1 signaling. PLoS One 4: e7124, 2009.

28. Lee SL, Son AR, Ahn J, et al: Niclosamide enhances ROS-mediated cell death through c-Jun activation. Biomed Pharmacother 68: 619-624, 2014. 